\title{
La question et la requête. Analyse sociopragmatique comparative dans le domaine français / roumain
}

\author{
Valentina Barbu \\ Université de Provence, Aix-Marseille I, en cotutelle avecl'Université de Bucarest \\ valentinalbarbu@gmail.com
}

\section{Introduction}

Cette communication a comme objectif principal d'apporter une réflexion sur la réalisation de deux actes de langage, la question et la requête, dans un contexte précis - l'incipit de l'interaction didactique. Ces actes sont relevés dans un corpus constitué de plusieurs classes de langue maternelle (français vs roumain), soit environ deux heures d'enregistrements pour chacune des langues analysées. Un objectif secondaire est de montrer un exemple d'analyse sociopragmatique comparative des deux actes directifs, réalisés en langues différentes.

Après une partie introductive dans laquelle on définit le cadre théorique de la présente étude, une deuxième partie traite des principales réalisations linguistiques des actes de langage susmentionnés, produits par les enseignants dans le cadre de l'interaction didactique. Enfin, une troisième partie propose une synthèse comparative des réalisations linguistiques dans le domaine français / roumain.

\section{Cadre méthodologique}

Nous nous situons dans le cadre de l'analyse des données " naturelles »" qui, de par leur caractère complexe, nécessitent une approche éclectique. On se contentera ici de mentionner l'existence des outils qui relèvent de l'analyse du discours ${ }^{2}$, de la pragmatique (théorie des actes de langage ${ }^{3}$ et pragmatique contrastive) et des courants interactionnistes (l'analyse conversationnelle ${ }^{4}$ et microsociologie : théorie des face-work ${ }^{5}$ ). Par ailleurs, nous rejoignons Kerbrat-Orecchioni (2005: 21) qui motive une telle démarche par souci de complétude pragmatique:

\begin{abstract}
$\mathrm{Au}$ lieu de s'épuiser en polémiques stériles (et qui ont parfois des allures donquichottesques, s'inventant un ennemi largement imaginaire afin de le mieux pourfendre), et d'opposer par exemple analyse de discours, analyse conversationnelle, théorie des actes de langage et théorie du face-work, il me semble plus intéressant et rentable de concilier ce qui est conciliable, et de voir le parti que l'on peut tirer du croisement de propositions provenant de paradigmes différents.
\end{abstract}

Avant de continuer la présentation de cette analyse, nous donnerons une description des termes-clé de la présente étude: il s'agit d'un côté de l' «acte de langage » et de l'autre côté de la situation de communication dans laquelle il est produit - l'incipit de l'interaction didactique.

Dans notre recherche, l' «acte de langage » est un concept souple qui intervient à deux niveaux de représentation: la communication et le discours. Ces deux acceptions du terme ne s'excluent pas réciproquement en dépit des polémiques plutôt stériles qui ont eu lieu entre les tenants de la théorie des speech acts d'un côté et ceux de l'analyse du discours de l'autre ${ }^{6}$. Il existe un cadre intégrant ces deux niveaux et il a été formalisé au sein du Groupe de Recherches sur les Communications de Nancy II par certains chercheurs, notamment Trognon\&Brassac (l'article de 1992 en offre une synthèse). En prenant comme point de départ la logique illocutoire développée par Searle\&Vanderveken (1985) et plus tard par Vanderveken seul $(1988,1990)$ qui prend comme composants primitifs non pas les actes de langage mais les composant de la force illocutoire, le modèle ainsi développé permet de dériver un ensemble de relations 
logiques articulant les propriétés des actes de langage. La logique illocutoire de la théorie des speech acts, entendu comme logique des relations entre réussite et satisfaction entre les actes de langage, n'est pas prise à la lettre dans le modèle susmentionné. D'un côté, les tenants de ce modèle étendent la portée de certaines lois de cette logique et de l'autre elle est interprétée dans un modèle dialogique face au modèle monologique initial.

Comme le déroulement d'une séance de langue maternelle est très complexe et diffère d'une langue à l'autre, nous nous sommes arrêtés sur un moment particulièrement riche du point de vue interactionnel, social et didactique, à savoir l'incipit, dans le but de donner une meilleure description du fonctionnement des actes de langage. L'enjeu est de dépasser la description pour mettre en évidences des régularités et des phénomènes qui puissent être utiles par la suite aux linguistes et / ou aux didacticiens. Ainsi l'incipit ${ }^{7}$ de l'interaction didactique est-il un concept que nous proposons pour nommer le début de l'interaction en classe entre l'enseignant et les élèves. Pour marquer les limites initiale et finale de ce moment dans le cadre du déroulement de l'interaction en classe $^{8}$, nous mentionnons la présence des indicateurs de nature différente. ${ }^{9}$ En effet, la limite initiale est marquée par des signaux de nature non-verbale (d'un côté on mentionne la sonnerie et de l'autre le regard du professeur posé sur tous les élèves ou sur certains d'entre eux) et verbale (des rappels à l'ordre, le plus souvent). La limite finale est, elle aussi, signalée par des indicateurs de nature non-verbale (le regard du professeur accompagné souvent de certains gestes), verbale (des requête qui demande de se mettre au travail) et didactique (l'incipit finit au moment ou le professeur a fini d'expliquer la première tache scolaire à résoudre pendant le cours en question. Plus précisément, le cours de RLM comporte deux moments dans le cadre de l'incipit : l'organisation de la classe et la vérification des devoirs. Le cours de FLM comporte lui aussi deux moments, mais pas les mêmes : le moment organisationnel et «Qui de neuf? » ou « Les news ».)

\section{Réalisations linguistiques des actes étudiés}

Suite à l'analyse du corpus enregistré et transcrit, nous avons constaté que les actes de langage qui apparaissent dans l'incipit de l'interaction didactique font partie de trois classes d'actes illocutoires d'après la classification de Searle (1972): les assertifs, les directifs et les expressifs. Pour la présente analyse nous avons choisi seulement deux actes de la classe des directifs, mieux représentés du point de vue quantitatif et qui regroupent des actes de langage qui ont à la base la valeur illocutoire de «demande ». Il s'agit da la « demande d'un dire » et de la «demande d'un faire », termes qui montrent déjà que la frontière entre ce que nous appellerons par la suite, question et respectivement requête, est indécise dans certains cas. Pour ce qui est des acceptions de ces actes, nous nous situons dans la lignée théorique de Kerbrat-Orecchioni (2001 : 86) par rapport à la question qui est définie comme « tout énoncé qui se présente ayant comme finalité principale d'obtenir de son destinataire un apport d'information.» En ce qui concerne la requête, nous la comprenons comme tout énoncé produit par un locuteur pour demander à son interlocuteur d'accomplir un acte quelconque $^{10}$.

\subsection{La question}

\subsubsection{Critères d'analyse}

Pour mettre un peu d'ordre dans la description d'un tel acte, nous nous sommes inspirés librement des critères exposés par Kerbrat-Orecchioni (2001 : 86-87) dans son ouvrage sur les actes de langage:

- Le locuteur ignore la réponse (questions proprement dites ${ }^{11}$ ) vs le locuteur veut vérifier si l'interlocuteur possède l'information demandée (questions didactiques). 
- L'information demandée concerne la valeur de vérité du contenu propositionnel global (questions totales) ou l'information demandée concerne un seul des constituants de la phrase (questions partielles).

- «La nature particulière de l'information demandée, et corrélativement, la fonction conversationnelle de la question »: questions régulatrices, demandes de clarification ou de reformulation, demander de confirmer vs infirmer, questions préliminaires, questions rituelles, questions absurdes ou ludiques, questions rhétoriques, questions pièges, questions alternatives, etc.

Les trois premiers critères groupés pour une première description donnent déjà une classification primaire de cet acte de langage, comme on le remarque dans les exemples ci-dessous, extraits du corpus analysé:

- Ex. (1) (FLM) Tu as fait les fiches ou pas ? (question proprement dite totale, alternative)

- Ex. (2) (RLM) A primit cineva două foi ? (fr. "Quelqu'un aurait reçu deux fiches?») (question proprement dite totale)

- Ex. (3) (FLM) Sixtine, combien est-ce qu'il y des mois dans l'année ? (question didactique partielle)

- Ex. (4) (RLM) Care este titlul, Alexandra? (fr. «Quel est le titre, Alexandra ?») (question didactique partielle)

Le dernier critère est aussi le plus complexe :

- Les marqueurs de la valeur illocutoire de la question

Par la suite, nous allons nous arrêter davantage sur ce critère qui nous donne des informations plus précises sur les réalisations linguistique et pragmatique de la question.

Avant de passer à l'analyse, il faut mentionner que la valeur de question peut être exprimée directement ou indirectement. Dans le domaine français / roumain, cette valeur est exprimée surtout directement à quelques exceptions près que nous signalerons par la suite.

Le premier cas de figure - la valeur de question est exprimée directement - se réfère aux énoncés possédant une structure interrogative due à la présence des marqueurs spécifiques de nature lexicale, morphosyntaxique, syntaxique ou/et prosodique. Nous passerons en revue les réalisations linguistiques des questions en fonction des marqueurs mentionnés ci-dessus.

Tout d'abord, on note l'absence de formule performative dans les deux langues étudiées, donc l'absence du marqueur de nature lexicale. Nous signalons la même situation pour les marqueurs de nature syntaxique ${ }^{12}$.

Ensuite, les marqueurs de nature morphosyntaxique sont présents dans les deux langues en grand nombre. En ce qui concerne la distribution syntaxique, les marqueurs de ce genre peuvent apparaître en position initiale, médiane ou finale. Le nombre élevé d'occurrences des marqueurs de ce type en début d'énoncé nous montre une préférence pour ce type de distribution.

Ainsi notons-nous en français la présence de la périphrase interrogative «est-ce que » pour les questions totales, et des pronoms, adjectifs ou adverbes interrogatifs tels que « qui », « quel(le) », « combien », " où », «comment», «pourquoi» ou «quoi» pour les questions partielles. Toujours pour ce dernier type de questions, on signale aussi la présence en début d'énoncé de la périphrase interrogative « qu'est-ce que ». En outre, dans certaines questions catégorielles, comme dans l'exemple (6) ci-dessous, on retrouve deux éléments interrogatifs, dont un de la série des périphrases interrogatives, signe d'un niveau de langue soignée.

Dans le corpus roumain, pour ce qui est des questions partielles, on remarque la présence d'un élément de la série des pronoms, adjectifs ou adverbes interrogatifs tels que « ce » (fr. « quoi »), « cum» (fr. « comment»), 
« care » (fr. « quel(le) »), « cine » (fr. « qui »), « când » (fr. « quand ») et « unde » (fr. « où »). Il n'y a pas de marqueur morphosyntaxique pour les questions totales dans le corpus roumain.

- $\quad$ Ex. (5) (FLM) Est-ce que les malades sont revenus ? (question totale)

- Ex. (6) (FLM) Où est-ce qu'il est allé ? (question partielle)

- Ex. (7) (RLM) Cine n-a citit ? (fr. « Qui (est-ce qui) n'a pas lu ?») (question partielle)

La deuxième situation - les marqueurs de nature morphosyntaxique en position médiane - représente un type de distribution syntaxique peu usitée tant par le français que par le roumain dans le corpus étudié.

- Ex. (8) (FLM) C'est l'histoire de quoi 'Oscar'?

- Ex. (9) (RLM) Altcineva cum a răspuns ? (fr. «Quelqu'un d'autre, comment a-t-il répondu » ?)

Pour conclure, nous notons que la distribution des marqueurs morphosyntaxiques en ce qui concerne la fréquence est semblable dans les deux langues. Ainsi l'extraposition à gauche enregistre-t-elle le plus d'occurrences. Les deux autres types de distribution apparaissent rarement. Par ailleurs, les distributions syntaxiques finale et médiane n'apparaissent que dans les questions partielles. En outre, nous remarquons que la présence de ces types de marqueurs, surtout s'ils se trouvent en fin d'énoncé, est liée à la demande des précisions. De plus, c'est une situation qui étaye l'idée que certaines valeurs illocutoires ne peuvent pas être séparées de leur contexte linguistique et situationnel. Ce n'est qu'en reconstituant l'échange professeur-élève qu'on a accès à la signification de ces énoncés.

- Ex. (10) (FLM) L'élève : Hier elle avait dit qu'elle l'avait mordue. / Le professeur : Mais elle vous a mordu où ?/ L’élève : Au bras.

- Ex. (11) (RLM) L'élève : Furnicile cântă deoarece le ajută mult la spor. (fr. Les fourmis chantent parce que cela les aide beaucoup à réaliser des bénéfices. ») Le professeur : La spor în ce ? (fr. « Des bénéfices dans quoi ? »)

Par ailleurs, on doit aussi signaler les éléments interrogatifs qui forment un énoncé à eux seuls :

- Ex. (12) (FLM) Pourquoi?

- Ex. (13) (RLM) Cine ? (fr. «Qui »)

Après les marqueurs de nature lexicale, syntaxique et morphosyntaxique, il est temps enfin de parler des marqueurs de nature prosodique ${ }^{13}$. Ceux-ci jouent un rôle très important pour la valeur illocutoire de question. Dans le corpus analysé nous avons décelé les situations suivantes :

- $\quad$ il y a des questions qui ne sont pas marquées de point de vue prosodique (ex. 14 et 15)

- $\quad$ il y a présence des marqueurs prosodiques interrogatifs même s'il ne s'agit pas d'une question (ex. 16 et 17)

- le marqueur prosodique est le seul marqueur de la valeur illocutoire de question (ex. 18 et 19)

En ce qui concerne la fréquence, les questions qui font partie du deuxième cas de figure sont les plus nombreuses. Le meilleur exemple dans les deux langues est la présence des whimperatives, terme emprunté à l'anglais pour désigner une requête exprimée indirectement sous la forme d'une question. Le premier cas de figure est lui aussi représenté, mais dans un seul énoncé en français et dans quelques uns en roumain. Pour illustrer ces descriptions, regardons les exemples ci-dessous:

- $\quad \operatorname{Ex}(14)(\mathrm{FLM})$ Les fiches, tu les as faites. 
- $\quad \operatorname{Ex}(15)$ (RLM) Ia să vedem cine şi-a dat seama. (fr. «Voyons qui s'en est rendu compte »)

- $\quad$ Ex (16) (FLM) Tu attends quoi, Louis?

- $\operatorname{Ex}(17)$ (RLM) Acolo e locul tău ? (fr. « Elle est là ta place? »)

- Ex. (18) (FLM) Tu les as apportés ?

- Ex. (19) (RLM) Ați mai avut şi altceva? (fr. « Vous avez eu quelque chose d'autre à préparer? »)

Nous voudrions nous attarder davantage sur les exemples que nous venons de donner car leur caractérisation est plus complexe qu'on pourrait le croire à première vue. En effet, un énoncé comme celui de l'exemple (14) serait interprété comme une situation de «superposition d'actes» par Fontaney (1991: 157) pendant que l'hypothèse de Kerbrat-Orecchioni (1991: 109) serait qu'il s'agit d'un continuum entre la question et l'assertion, donc d'un statut intermédiaire. Quant à nous, l'analyse du contexte d'énonciation nous montre qu'effectivement dans cet énoncé il y a une coalescence des deux actes (la complexité du schéma prosodique le montre aussi) et que l'un des deux, en l'occurrence la question, sera satisfait dans l'intervention suivante (conformément à la logique illocutoire : Searle\&Vanderveken, 1985). De l'autre côté, les actes qui précédent l'énoncé en question nous donne des indices en faveur de l'interprétation de cet énoncé en tant qu'assertif (du moins, en ce qui concerne l'intention du professeur).

- $\operatorname{Ex}\left(14^{\prime}\right)$

Le professeur: Ah! Tu me le donnes alors? Le commissaire et la sorcière. Tu me le donnes alors? Tu l'as fait. Les fiches tu les as faites.

Les élèves : Non.

Un autre type d'énoncé qui poserait problème est illustré dans l'exemple (15). En outre, dans l'interaction didactique roumaine, nous avons identifié neuf énoncés de ce type. Le problème qui se pose est toujours lié à l'appartenance : s'agit-il de la classe des questions ou de la classe des requêtes ? Ces énoncés ont la même structure syntaxique: $\mathrm{Ph}_{1}$ (ia să vedem « voyons ») $+\mathrm{Ph}_{2}$ (qui est une question identifiée comme telle grâce aux marqueurs morphosyntaxiques). La prosodie est spécifique de l'assertion. Nous postulons que formellement il s'agit de deux actes de langage différents - une assertion et une question - qui se suivent linéairement. Au contraire, du point de vue pragmatique, il s'agit d'une question, l'acte satisfait « suivant la direction d'ajustement propre à son but illocutoire » (Vanderveken, 1988: 134). En effet, le mode d'ajustement est du monde aux mots car les élèves donnent l'information attendue. La question est satisfaite conformément aux relations logiques entre cet acte et les autres qui le suivent, mais que nous ne reproduirons pas ici faute d'espace.

Le deuxième cas de figure - la valeur de question exprimée indirectement - est moins représenté de point de vue de la fréquence. Cette valeur vient se greffer sur un autre acte de langage. Dans son ouvrage sur les actes de langage, Kerbrat-Orecchioni (2001) recense trois cas de figure relevant de la formulation indirecte conventionnelle de la question, à savoir:

- $\quad$ assertion du but illocutoire de la question

- question portant sur la condition de réussite relative au destinataire

- $\quad$ assertion portant sur la condition de réussite concernant le locuteur.

Suivant cette description, nous avons trouvé un énoncé relevant du deuxième cas de figure, pour le français, et un énoncé relevant du premier cas, pour le roumain :

- $\quad$ Ex. (20) (FLM) Vous savez pourquoi il est pas là Paul ? 
- Ex. (21) (RLM) Ia să vedem care este titlul. (sous-entendu: “Care este titlul ?”) (fr. « Voyons quel est le titre. »; sous-entendu: « Quel est le titre »?)

\subsubsection{Enchaînement et orientation de la réponse}

On le sait bien, la question est un acte par excellence initiatif. En règle générale, elle est suivie d'une réaction $^{14}$. Dans le cadre des interactions didactiques analysées, la question est suivie soit d'une réaction verbale soit d'une réaction non verbale. En classe de FLM, les questions « orientent » les réponses soit par des marqueurs sémantiques («avoir envie de », «croire que») en cooccurrence avec des marqueurs morphosyntaxiques (la périphrase verbale «est-ce-que»), soit par des marqueurs prosodiques (ex. 13). Pareillement, en classe de RLM, les marqueurs qui « orientent» les questions sont de nature sémantique et prosodique, à la seule différence qu'en roumain il n'y a pas de marqueurs morphosyntaxiques qui joueraient ce rôle. Par ailleurs, nous postulons que les réponses sont aussi orientées par le spécifique de l'interaction analysée. Le jeu des rôles, des statuts et des savoir-faire dans le cadre de l'interaction didactique influencent les réalisations linguistiques des actes de langage en contexte authentique.

- Ex. (22) (FLM) Le professeur : Est-ce que vous avez envie d'apprendre ? ou encore

- Le professeur : Est-ce que tu crois que quand je donne un travail comme ça ben tous les enfants peuvent le faire quand ils veulent ce travail-là?

- $\quad$ Ex. (23) (FLM) Toute la journée ? (question rhétorique)

- $\quad$ Ex. (24) (RLM) Le professeur : Unde suntem noi atunci când se sună ? ( fr. « Où sommes-nous quand la sonnerie retentit »?)/ Les élèves : În classă ! (fr. « En classe !»)

- Ex. (25) (RLM) Doar tu eşti în clasa asta, Raluca? (fr. « Il n’y a que toi dans cette classe, Raluca ?»)

\subsection{Réalisations linguistiques de la requête}

Comme nous l'avons annoncé, nous continuons l'étude avec l'analyse des réalisations linguistiques de la requête. Tout comme dans le cas de la question, cette analyse suivra deux volets: réalisations directes et réalisations indirectes.

Premièrement, dans le cadre des formulations directes ${ }^{15}$ en français comme en roumain, nous mentionnons l'existence des énoncés à l'impératif:

- Ex. (26) (FLM) Prends ton crayon à papier!

- Ex. (27) (RLM) Stai jos! (fr. « Asseye-toi!»)

L'étude parallèle de quelques énoncés roumains et de leurs traductions françaises met en évidence comme procédés de transcodage les plus fréquents les traductions directes: les interjections roumaines à valeur exhortative, « hai să » et « ia să ${ }^{16}$ », sont rendues en français par des verbes à l’impératif:

- Ex. (28) Hai să deschidem să citim lectura «Toamnă ». (fr. " $\underline{\text { Allez}}$, on va ouvrir le livre on va lire la lecture « Automne ».)

- Ex. (29) Ia să vedem ce temă am avut pentru astăzi. (fr. « $\underline{\text { Voyons }}$ donc ce qu'on a eu comme devoir pour aujourd'hui. ») 
Deuxièmement, en ce qui concerne les formulations indirectes des requêtes, celles-ci sont nombreuses, en français comme en roumain. Catherine Kerbrat-Orecchioni fait une synthèse des critères de classements des formulations indirectes (op.cit., 99-105) que nous reprenons dans notre étude:

Tout d'abord, d'après le critère «types d'actes de langage 'squattés' par la requête, et détournés à son profit » (idem), on remarque la présence des questions à valeur de requête (angl. whimperatives) comme dans les exemples (30) et (31), mais aussi des assertions à valeur de requête ${ }^{17}$ comme nous le montre les exemples (32) et (33):

- $\quad$ Ex. (30) (FLM) Tu donnes ça, s'il te plaît, Baptiste?

- Ex. (31) (RLM) Toată lumea a deschis cartea ? (« Tout le monde a ouvert le livre?»)

- Ex. (32) (FLM) Je préfère un autre. (sous-entendu : « Dis-moi un autre. »)

- Ex. (33) (RLM) Nu avem nevoie de penar. (fr. «On n'a pas besoin de trousse ${ }^{18}$.» sous-entendu: « Remets ta trousse. »)

Nous considérons que les whimperatives forment une catégorie à part, intermédiaire, entre la question et la requête, du moins dans le domaine français / roumain de l'interaction didactique. Il s'agit d'une structure additive dans laquelle les deux actes se trouvent plutôt en relation de coalescence (voir supra " superposition d'actes $»)^{19}$. En outre, ces actes de langage sont toujours « orientés ». Dans le corpus roumain, les marqueurs ayant la fréquence la plus élevée, ceux de nature sémantique, forment un groupe où chaque énoncé contient le mot $d a$ à intonation ascendante (fr. « d'accord » ou «n'est-ce pas ») comme dans l'exemple ci-dessous :

- Ex. (34) Bănuiesc că v-ați pus-o la portofoliu, da ? (fr. « Je suppose que vous l'avez mise dans le porte vue, n'est-ce pas?»)

Ensuite, en fonction du deuxième critère, «le caractère plus ou moins conventionnel de l'acte indirect », on remarque qu'il y a des tournures qui fonctionnent en français ou en roumain comme des requêtes indirectes. La langue française admet les formes suivantes de requêtes indirectes conventionnelles («tropes lexicalisés $\gg{ }^{20}$ ):

- «structure interrogative à la deuxième personne, comportant le modalisateur ' pouvoir ' ou ' vouloir ' à l'indicatif ou au conditionnel» (ex. 35)

- « question sur la possibilité qu'à le destinataire de fournir cet objet» (ex. 36)

- «assertion à la première personne comportant les verbes 'aimer (bien)' au conditionnel, ou 'vouloir' à l'indicatif ou au conditionnel» (ex. 37).

- Ex. (35) Tu veux nous la montrer ? ou encore Alors les facteurs vous pouvez $[\ldots]^{21}$ ?

- $\quad$ Ex. (36) Ton tablier il est où, Baptiste ? (sous-entendu : « Mets ton tablier ! »)

- $\quad$ Ex. (37) Je voudrais que tout le monde écoute.

Quoiqu'en roumain on puisse trouver les types de tropes énumérés ci-dessus, dans le corpus étudié nous n'en avons trouvé aucun.

Enfin, le dernier critère, «le caractère plus ou moins brutal ou au contraire adouci de la formulation » (Kerbrat-Orecchioni, 2001 :102) de la requête, met en évidence des formulations indirectes qui ont des allures très autoritaires, pouvant être considérés comme des ordres. Dans le corpus français, nous avons recensé les situations suivantes en ce qui concerne les énoncés les plus marqués de point de vue de la brutalité de la formulation : 
- des assertions en « je veux » (aucune occurrence)

- des assertions comportant un modalisateur déontique (à valeur d'obligation) (exemples 38 et 39)

- des assertions à l'indicatif, futur ou présent (exemples 40 et 41).

- $\quad$ Ex. (38) (FLM) Ensuite, il faut remettre les phrases en ordre.

- $\quad$ Ex. (39) (RLM) Trebuie să fim cuminți. (fr. « On doit être sages. »)

- Ex. (40) (FLM) Vous attrapez votre cahier de règles.

- Ex. (41) (RLM) Vom spune propoziții folosind cuvântul «muşuroi ». (fr. «On va dire des exercices avec le mot 'fourmilière'. »)

Par ailleurs, Renaud Camus, cité par Kerbrat-Orecchioni ${ }^{22}$ parle du fait que le mode impératif est de moins en moins utilisé de nos jours et qu'il est remplacé par l'indicatif qui n'est pas pour autant moins autoritaire. Effectivement, dans le corpus étudié, le nombre d'occurrences des énoncés aux verbes à l'indicatif est plus élevé que le nombre des énoncés aux verbes à l'impératif.

A la fin, nous parlerons de la présence des «adoucisseurs » et des «aggravateurs » dans la formulation de la requête. Ainsi les formules de politesse «je te/vous prie de » ou «s'il te plaît ${ }^{23}$ ", présentes dans le corpus roumain et le pronom «on » à valeur hypocoristique dans le corpus français, font partie de la première catégorie. Enfin, nous remarquons la présence de deux autres adoucisseurs, un de nature pragmalinguistique «demander l'accord sur un fait $»^{24}$, comme dans les exemples (42) et (43), et un de nature lexicale - les interjections injonctives d'exhortation "hai (să) + première personne du pluriel », "ia (să) + première personne du pluriel» en roumain (revoir les exemples (39) et $(41)^{25}$. A part la valeur d'adoucisseur, ces interjections marquent aussi le caractère familier de la formulation, aspect important pour la relation interpersonnelle.

- Ex. (42) (RLM) La pauza mare să-mi aduci aminte, da? (fr. «Pendant la grande récréation, tu me le rappelleras, d'accord ?»)

- Ex. (43) (FLM) Il faut juste que vous me le demandiez, d'accord?

Dans la catégorie des «aggravateurs», nous mentionnons la présence du fameux «ton », dans les deux langues étudiées. En outre, en français il y a aussi le cas de l'interjection injonctive d'exhortation.

En conclusion, on doit mentionner qu'en roumain comme en français, dans le corpus étudié, il y a des requêtes qu'on appellerait requêtes didactiques ${ }^{26}$ indirectes, des structures presque figées qui sont dépendantes du contexte didactique et qui n'apparaissent que dans ce contexte précis. Pour plus de précisions, regardons les exemples suivants :

- Ex. (44) (FLM) Préparez vos affaires! ou Rangez vos affaires ! ou Les CE1 !

- Ex. (45) (RLM) Lecția începe. (« On commence le cours. ») ou Clasa a II-a ! (Les CE1 !)

La requête, acte «menaçant » par excellence, est sans doute l'un des actes les plus étudiés par les linguistes, non seulement dans une perspective « intra-culturelle » mais aussi « cross-culturelle ${ }^{27}$.

\subsection{Quelques éléments de synthèse comparative}

L'analyse comparative des actes de langage directifs dans l'incipit de l'interaction didactique dans le domaine français/roumain nous montre une fois de plus que la thèse universaliste des actes de langage est une fausse piste. Certes, l'existence d'un grand nombre de similitudes dans les deux langues peut nous induire en 
erreur. Nous mentionnons d'abord le fait que les actes de langage de la classe des directifs qui apparaissent dans le moment susmentionné de l'interaction didactique sont la question et la requête. Nous n'avons trouvé aucun énoncé performatif à valeur de requête ou de question. Ensuite, la valeur illocutoire de requête peut être formulée de façon directe ou indirecte, brutale ou adoucie. En outre, on remarque l'existence des formulations indirectes comme la question et l'assertion à valeur de requête dans les deux langues étudiées. Toujours par rapport à la requête, nous mentionnons l'existence des adoucisseurs en français comme en roumain dans les formulations indirectes de cet acte de langage. Au contraire, les types d'adoucisseurs diffèrent d'une langue à l'autre comme nous le verrons infra. En outre, elle est toujours un acte initiatif et elle appartient toujours au professeur.

Lorsqu'on se situe dans la perspective contrastive, on remarque vite que l'opinion générale des linguistes selon laquelle les actes de langage ne sont pas conçus de la même manière dans deux langues différentes est vraie. Les facteurs de variation se trouvent surtout du côté des formulations, de l'enchaînement et de la fréquence.

Ainsi ferons-nous premièrement la synthèse des formulations différentes des deux actes de langage étudiés. Le premier volet de l'aspect linguistique porte sur les moyens morphosyntaxiques. Par rapport à la question, en français on rencontre des morphèmes et des périphrases interrogatives en début d'énoncé pour les questions totales et des pronoms et des adverbes interrogatifs pour les questions partielles. En revanche, en roumain on ne rencontre que des pronoms, des adjectifs ou des adverbes interrogatifs. Il n'y a pas de périphrase ou de morphème interrogatifs.

Enfin, en ce qui concerne le deuxième facteur de variation - la fréquence - nous indiquerons que le nombre des questions en français est beaucoup plus important qu'en roumain. En échange, les requêtes apparaissent dans les deux langues presque avec la même fréquence. En outre, en ce qui concerne le type de question, le nombre des questions didactiques est nettement supérieur par rapport aux questions proprement dites dans le corpus roumain que dans celui français. Et on remarque le phénomène inverse, c'est-à-dire dans le corpus français il y a plus de questions proprement dites que de questions didactiques.

Du point de vue culturel et didactique, il y a des énoncés qui montrent à quel point l'incipit acquiert aussi la valeur de «rituel pédagogique » spécifique d'une pratique pédagogique particulière, voire une philosophie éducative spécifique. Et nous pensons d'une part aux questions organisationnelles, et d'autre part aux requêtes didactiques indirectes, propres à l'interaction didactique et/ou au style pédagogique du professeur français étudié. Par ailleurs, en roumain, on rencontre un autre type de question à fort degré de standardisation linguistique et didactique. Il s'agit des questions qui visent à vérifier les devoirs écrits et oraux. Donc, les variations de la réalisation des actes de langage en français et en roumain ne sont pas uniquement de nature linguistique. Encore deux autres dimensions s'y rajoutent - culturelle et éducationnelle - dimensions qui constitueront le sujet d'une autre analyse. Et nous pensons que ce serait plus judicieux de parler plutôt de « culture linguistique » et $«$ culture didactique ${ }^{28}$ dans la réalisation de ces actes de langage.

\section{En guise de conclusion}

A travers de cette étude, nous avons essayé de jeter quelque lumière sur la réalisation de deux actes de langage directifs - la question et la requête - en contexte authentique d'interaction. L'étude empirique a été réalisée en fonction de trois approches principales: «intra-langue», sociopragmatique et «cross-culturelle». Les résultats ont mis en évidence d'un côté le fait que les frontières entre les deux actes de langage étudiés ne sont pas claires, et de l'autre côté la nécessité de prendre en compte plusieurs dimensions - linguistique, énonciative, culturelle et didactique - dans l'interprétation pertinente de leur force illocutoire. 


\section{Références bibliographiques}

Béacco, J.C., Chiss, J.L., Cicurel, F., Véronique, D. (dir.) (2005). Les cultures éducatives et linguistiques dans l'enseignement des langues, Paris : Presses Universitaires de France

Blum-Kulka, S. (1984). « Requests and apologies: a cross-cultural study of speech act realization patterns CCSARP » in Applied Linguistics, 5, p.196

Brassac, Ch. (1992). «Analyse de conversations et théorie des actes de langage » in Cahiers de Linguistique Française $13,62-75$

Comes, E. (2006). Interjections injonctives d'exhortation en roumain et en français in Araújo Carreira, M.H. (éd.), Travaux et documents, 32-2006, «Venez, venez! » De la suggestion à l'injonction dans les langues romanes, Paris : Université Paris 8, Vincennes-Saint-Denis , 75-89

Fontaney, L. (1991): « A la lumière de l'intonation », in Kerbrat-Orecchioni, C. (éd.) La Question, Lyon : PUL, 113-161

Frédéric, F. et al. (1990). La communication inégale. Heurs et malheurs de l'interaction didactique, Paris : Délachaux et Niestlé

Goffman, E. (1974). Les rites d'interaction, Paris : Les Editions de Minuit

Gumperz, J. J. (1989). Engager la conversation : introduction à la sociolinguistique interactionnelle, Paris : Les Editions de Minuit

Kerbrat-Orecchioni, C. (1990-1994). Les interactions verbales, tome I, II et III. Paris : Armand Colin

Kerbrat-Orecchioni, C. (1991) : «L'acte de question et l'acte d'assertion : opposition discrète ou continuum ? », in Kerbrat-Orecchioni, C. (éd.) La Question, Lyon : PUL

Kerbrat-Orecchioni, C. (2001). Les actes de langage dans le discours. Théorie et fonctionnement, "Quand dire c'est faire »: un travail de synthèse sur la pragmatique conversationnelle, Paris : Nathan

Kerbrat-Orecchioni, C. (2005). Le discours en interaction, Paris : Armand Colin

Martins-Baltar, M. (1976). «Actes de parole» in Un Niveau Seuil, Conseil de la Coopération Culturelle du Conseil de l'Europe

Mitchell, K. (1981). «Illocutionary acts in a pedagogical description - the grammar of requests and offers », in Richterich, R., Widdowson, H.G. (éds.): Description, présentation et enseignement des langues, Langues et apprentissage des langues (LAL), Paris: Hatier

Moeschler, J. (1985). Argumentation et conversation. Eléments pour une analyse pragmatique du discours, Paris : Hatier

Rabatel, A. (éd.) (2004). Interactions orales en contexte didactique. Mieux (se) comprendre pour mieux (se) parler et pour mieux ( $s^{\prime}$ ) apprendre, IUFM de L'Académie de Lyon, Lyon : Presses Universitaires de Lyon

Roulet, E. et al. (1985). L'articulation du discours en français contemporain, Berne : Peter Lang

Searle, J. R. (1972). Les actes de langage, Paris : Hermann

Searle, J. R. (1979). Sens et expression, Paris : Minuit

Searle, J.R., Vanderveken, D. (1985). Foundations of Illocutionary Logic, Cambridge: Cambridge University Press

Sinclair, J. Mch., Coulthard, R.M. (1975). Towards an Analysis of Discourse. The English used by teachers and pupils, Oxford University Press

Traverso, V. (1999). L'analyse des conversations, Paris : Nathan

Trognon, A., Brassac, C. (1992). « L’enchaînement conversationnel », Cahiers de Linguistique Française 13, 76-107

Vanderveken, D. (1988). Les actes de discours, Liège : Pierre Mardaga 
${ }^{1}$ «Données 'naturelles' »- traduction du syntagme anglais naturally occuring ou natural data. Dans notre recherche, pour la collecte de ce type de données, nous avons fait des enregistrements vidéo. Il s'agit de huit classes de langue maternelle pour chacune des deux langues, enregistrées et transcrites par nous même. Mais, comme nous avons choisi d'analyser seulement l'incipit de ces classes, l'analyse effective est faite sur 120 minutes cumulées.

${ }^{2}$ Il s'agit des représentants de l'Ecole de Genève, et nous prenons comme ouvrages de référence: Roulet, E. et al. (1985) et Moeschler, J. (1985)

${ }^{3}$ Les ouvrages de référence sont : Searle, J. R. (1972, 1979), Searle\&Vanderveken (1985) et Vanderveken, D. (1988)

${ }^{4}$ Kerbrat-Orecchioni, C. (1990-1994, 2001)

${ }^{5}$ Goffman, Ervin (1974)

${ }^{6}$ Pour plus de détails par rapport aux positions théoriques différentes de Searle et de Moeschler en ce qui concerne les actes de langage, voir Moeschler, J. (1996 : 195-206), mais aussi Brassac, Ch. (1992)

${ }^{7}$ Dans le domaine de l'analyse des interactions, on ne trouve que les termes «ouverture », « séquence d'ouverture » ou « préambule » mais jamais « incipit » pour décrire le début d'une interaction. Nous avons trouvé des descriptions plus ou moins sommaires des moments qui correspondraient partiellement au moment visé par notre étude, mais pas une seule description linguistique complexe.

${ }^{8}$ Dans cette étude, à la différence du deuxième modèle genevois (voir Roulet, et $a l$. 1985), nous avons utilisé un modèle à cinq rangs : l'interaction, la séquence, l'échange, l'intervention et l'acte de langage. Dans le modèle standard, les constituants de la conversation sont toujours au nombre de cinq, mais la terminologie diffère sensiblement de celle adoptée dans notre analyse, à savoir: l'incursion (de point de vue de l'extension celle-ci est identique à ce que nous appelons « le cours de langue maternelle »), la transaction, l'échange, l'intervention et l'acte de langage.

${ }^{9}$ Nous faisons la différence entre l'interaction qui a lieu dans la salle de classe, l'incipit de l'interaction didactique, et la pré-interaction qui commence dans la cour de récréation et qui prépare le moment qui nous intéresse. Nous postulons la présence des indicateurs de la co-présence dans les deux situations.

${ }^{10}$ En réalité, c'est la même description que celle de Kerbrat-Orecchioni (2001: 98), à la seule différence que nous considérons que les actes à accomplir par l'interlocuteur peuvent être aussi de nature verbale (celle-ci ne constitue pas un apport d'information comme dans le cas de la réponse à une question).

${ }^{11}$ Là encore nous avons quelques remarques à faire par rapport aux positions théoriques des linguistes face à ces catégories. Kerbrat-Orecchioni n'utilise pas le terme de «question proprement-dite». Nous l'avons emprunté à Searle pour faire la différence entre ce type de question et les questions didactiques qui abondent dans l'interaction en classe. Encore une précision terminologique: de notre point de vue les questions d'examen représentent une sous-classe des questions didactiques.

${ }^{12}$ En français on ne retrouve pas l'inversion du pronom sujet et en roumain il n'y a pas de marqueur de nature syntaxique qui puisse signaler qu'il s'agit d'une question, comme en français.

${ }^{13}$ En fait, on devrait parler des marqueurs de nature prosodique et typographiques. Mais, comme les interactions analysées sont exclusivement orales, nous avons préféré parler seulement des marqueurs prosodiques. Nous avons mis des points d'interrogation et d'autres marqueurs de nature typographique pour rendre les exemples plus "compréhensibles ». Habituellement, nous n'avons pas besoin de mettre de tels signes pour marquer les valeurs illocutoires des actes de langage, cette chose devenant explicite à l'aide du système de transcription que nous utilisons pour la collecte et l'analyse des données, à savoir HIAT décrit par Norbert Dittmar (Université Libre de Berlin) et EXMaRALDA une application de ce programme dont le concepteur est Thomas Schmidt (Université de Hambourg). Ce système sous forme de partition nous permet d'avoir une ligne pour marquer le verbal, une deuxième pour le paraverbal et une troisième pour le non verbal, pour chaque participant à l'interaction, ce qui nous donne une image détaillée de la réalisation de chaque acte. 
${ }^{14}$ Kerbrat-Orecchioni $(2001: .92)$ distingue les « réponses » des « répliques ». Dans le cas de l'interaction didactique, il n'y a pas de réplique dans le sens de l'auteur. En plus, la situation où l'interlocuteur refuse de répondre est très peu probable. De par son statut, l'élève ne peut ni « répliquer », ni refuser de répondre. Certes, il y a des exceptions.

${ }^{15}$ On parle de réalisation directe d'un tel acte de langage soit s'il est réalisé par une formule explicitement performative soit s'il recourt au mode impératif. On doit mentionner l'absence de formule performative explicite dans les deux langues.

${ }^{16}$ Pour une étude plus approfondie de ce type d'interjections, voir Comes, E. (2006 : 76-89)

${ }^{17}$ A cette même occasion nous voudrions signaler qu'en général il serait plus judicieux de parler de « requête qui est exprimée formellement par un 'énoncé déclaratif/ interrogatif' (cf. Vanderveken, 1988: 22)» que de dire « assertion/question à valeur de requête ». Des formules de ce type peuvent prêter à confusion. Selon nous, il est important de faire la distinction entre la « force illocutoire » d'un acte de langage et sa « réalisation linguistique » aussi au niveau du métalangage. La remarque tient la route tant que nous ne sommes pas confrontés à une situation du type « superposition d'actes » où on peut soutenir que les deux actes coexistent et il n'yen a pas un qui substitue l'autre (le cas de figure nommé par Kerbrat-Orecchioni « trope illocutoire »).

${ }^{18}$ Pour comprendre un tel énoncé, il faudrait voir ce qui se passe entre les participants à l'interaction. Comme notre idée de départ est que certaines valeurs illocutoires ne peuvent pas être comprises que si on étude les actes de langage dans leur contexte énonciatif, nous avons choisi de faire des enregistrements. Dans ce cas précis, un des élèves avait sorti sa trousse et c'est à ce moment que le professeur dit « On n'a pas besoin de trousse », ayant le sous-entendu « Remets ta trousse ». Grâce aux images et au contexte linguistique (car avant le professeur avait expliqué une tâche scolaire), on saisit la vraie valeur illocutoire d'un énoncé en dépassant la structure formelle.

${ }^{19}$ Effectivement, si on regarde de plus près les exemples (30) et (31), et surtout leurs conditions de réussite et de satisfaction dans leur contexte d'énonciation, que nous ne pouvons pas reproduire ici faute d'espace, on remarque le fait que les requêtes sont satisfaites mais que leur réussite nécessite le passage par la satisfaction des questions.

${ }^{20}$ Ces formes sont recensées par Kerbrat-Orecchioni dans son ouvrage sur les actes de langage (2001: 100-101)

${ }^{21}$ Là encore il s'agit de l'importance de la vidéo. Dans ce cas précis l'acte directif comporte un côté verbal et un côté non verbal. En effet, l'acte verbalisé s'arrête sans laisser l'impression qu'il autre chose à dire. Par contre il continue par un geste (la main tendue du professeur qui donne quelques fiches aux élèves sollicités) qui donne des indications sur la valeur illocutoire : il ne s'agit pas d'une question, mais d'une requête.

${ }^{22}$ En outre, nous reproduisons un fragment de son analyse: « Ce qui nuit à l'impératif, peut-être, c'est son caractère trop impératif, justement. On pense qu'un autre mode sera plus doux. Mais l'indicatif est bien plus autoritaire encore, puisque l'ordre donné et son exécution coöncident exactement en une seule performance verbale, comme si l'interlocuteur n'avait aucune volonté propre, pas un mot à dire, existait à peine ».

${ }^{23}$ Il nous semble très intéressant de noter le fait que ces formules, présentes dans quatre énoncés, apparaissent en tout début d'interaction, ce qui joue un rôle très important pour la relation interpersonnelle car elles font en amont un travail de face-work. Ces formules adoucissent le caractère menaçant de la requête.

${ }^{24}$ En réalité, il s'agit d'une fausse demande d'accord.

${ }^{25}$ En français l'interjection exhortative d'injonction «allez » peut apparaître comme un adoucisseur si elle est doublée d'une certaine intonation qui exprime plutôt une prière qu'un ordre. Mais ce n'est pas le cas dans notre corpus.

${ }^{26}$ Certaines requêtes peuvent être considérées comme étant «spécifiques de l'interaction didactique » et nous allons les appeler par la suite «requêtes didactiques». Plus que cela, il y en a quelques unes propres à l'incipit de l'interaction didactique.

${ }^{27}$ Comme le témoigne les résultats du CCSARP (Blum-Kulka, 1984)

${ }^{28}$ Cf. Béacco, J.C., Chiss, J. L., Cicurel, F., Véronique, D. (éd.) (2005 : 268-276) 